\title{
COVID-19: do enfrentamento ao fortalecimento de estratégias em saúde mental -
}

\section{uma revisão narrativa}

\author{
Daniela Sousa de Oliveira ${ }^{1}$ \\ Alan Cristian Firmo ${ }^{2}$ \\ Iony Cunha Bezerra ${ }^{3}$ \\ José Helder Campos Leite ${ }^{4}$
}

1.Terapeuta Ocupacional Residente em Saúde Mental do Adulto, Escola Superior de Ciências da Saúde- ESCS/FEPECS.

2.Farmacêutico Residente em Saúde Mental do Adulto, Escola Superior de Ciências da Saúde- ESCS/FEPECS.

3.Assistente Social Residente em Saúde Mental do Adulto, Escola Superior de Ciências da Saúde- ESCS/FEPECS.

4.Fisioterapeuta Residente em Saúde Mental do Adulto, Escola Superior de Ciências da Saúde- ESCS/FEPECS.

Endereço para correspondência: aleinda21@gmail.com

\section{RESUMO}

A pandemia exige das autoridades respostas imediatas, para que se possam traçar linhas de cuidados e, assim, reagir aos enfrentamentos e, desse modo, elaborar estratégias que poderão nortear as intervenções. $\mathrm{O}$ isolamento social tem ocasionado diversos tipos de problemáticas relacionadas à saúde mental da população e dos trabalhadores da saúde. Com isso, o objetivo deste estudo é verificar as informações veiculadas por autoridades sobre a saúde mental e a COVID-19. Optou-se pela revisão de narrativa em bancos de dados, sites oficiais de estados do Brasil e portais de pesquisa. Os resultados expressam tentativas de alinhamento das normativas às recomendações sobre os cuidados com saúde mental no período de pandemia da OMS. Nota-se a variedade de dispositivos de divulgação e utilização de notas informativas aos profissionais e gestores da saúde. Foram poucos os estados que se preocuparam com rede psicossocial ativa, especificamente no sentido de propor melhorias. Considera-se a importância de instrumentalização dos gestores, trabalhadores e usuários do SUS, com o intuito de elaborar estratégias no enfrentamento à COVID-19 que permita a participação popular na tomada de decisão.

Palavras Chaves: Covid-19, saúde mental, isolamento social 
COVID-19: from coping to strengthening mental health strategies - a narrative

review

\begin{abstract}
The pandemic demands immediate responses from the authorities, so that lines of care can be drawn and, thus, react to the confrontations and, thus, develop strategies that may guide the interventions. Social isolation has caused several types of problems related to the mental health of the population and health workers. The objective of this study is to verify the information provided by authorities about mental health and COVID-19. We opted for reviewing narrative in databases, official websites of Brazilian states and research portals. The results express attempts to align the norms with the recommendations on mental health care in the WHO pandemic period. Note the variety of devices for the dissemination and use of information notes to health professionals and managers. Few states were concerned with an active psychosocial network, specifically in the sense of proposing improvements. It is considered the importance of instructing SUS managers, workers and users, in order to develop strategies to face COVID-19 that allows popular participation in decision making.
\end{abstract}

Keywords: covid-19, mental health, social isolation

\title{
INTRODUÇÃO
}

Em dezembro de 2019, um grupo de pacientes diagnosticados com pneumonia de etiologia ainda desconhecida, foi identificado em Wuhan, na China. As autoridades chinesas confirmaram que um novo coronavírus havia sido o patógeno causador, o qual se espalhou rapidamente em todas as províncias da China, sendo disseminado para mais de 40 países. O novo coronavírus pertence a uma família de vírus que pode desencadear doenças respiratórias em humanos e foi nomeado de severe acute respiratory syndrome coronavirus 2 (SARS-CoV-2) pela Organização Mundial da Saúde (OMS). A doença causada por este patógeno foi denominada COVID-19 ${ }^{1}$.

Em cerca de três meses, desde o início da epidemia na China, já ocorreram mais de 400 mil casos no mundo e 19 mil mortes; estima-se que esses números podem aumentar no decorrer dos meses ${ }^{2}$. Enquanto isso, no Brasil, até o dia 20 de abril de 2020, 
já foram registrados 40.581 casos e 2.575 óbitos por COVID-19, onde a maior parte dos casos notificados se concentram nos estados de São Paulo e Rio de Janeiro ${ }^{3}$.

Os coronavírus são considerados potencialmente patogênicos por desencadear infecções respiratórias em seres humanos, as quais podem variar de resfriados comuns a condições clínicas mais graves, tais como a Síndrome Respiratória Aguda Grave (SARS) e a Síndrome Respiratória do Oriente Médio (MERS) ${ }^{1}$. Os sintomas da infecção por SARS-CoV-2 aparecem após um período de incubação de aproximadamente 5 dias, o qual depende da idade e do sistema imune do paciente. As manifestações clínicas da infecção por SARS-CoV-2 são amplas e podem variar desde um simples resfriado até uma pneumonia grave. Os sinais e sintomas clínicos referidos são principalmente os respiratórios, de modo que o paciente pode apresentar "febre, tosse e dificuldade para respirar, enquanto outros sintomas inespecíficos incluem fadiga, mialgia, artralgia, dor de

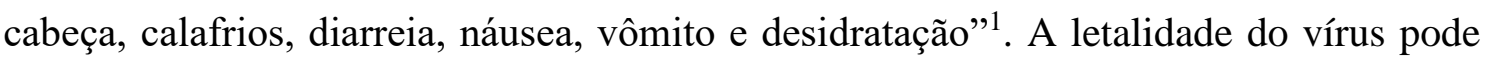
variar conforme a faixa etária e caso o paciente apresente condições clínicas associadas (diabetes, hipertensão, problemas respiratórios, etc) ${ }^{1}$. A COVID-19 tem alta transmissibilidade, de modo que a transmissão ocorre pelo contato direto ou por gotículas espalhadas pela tosse ou espirro de um indivíduo infectado. A transmissão pode ocorrer mesmo que o indivíduo não apresente os sinais e sintomas da doença ${ }^{1}$.

Para controlar a disseminação do vírus é necessário implementar medidas de prevenção e controle, tais como a lavagem de mãos com água e sabão ou álcool em gel, cobrir a boca e nariz ao espirrar ou tossir, se manter em ambiente arejado e evitar aglomerações ${ }^{4}$. O isolamento social é uma das estratégias para minimizar a propagação do vírus e evitar a sobrecarga do sistema de saúde, em decorrência do aumento do número de casos por COVID-19. No entanto, estudos mostram que a desconexão social com 
familiares e amigos tende a aumentar o risco de depressão e ansiedade, gerando consequências físicas e mentais para a saúde da população ${ }^{5}$.

Em um contexto de pandemia, as atenções e esforços se voltam, naturalmente, às especificidades de saúde relacionadas ao agente patogênico em questão, no entanto, sabese que também a saúde mental pode ser afetada das mais diversas formas ${ }^{6}$. A incerteza quanto ao futuro; a apreensão acerca dos acontecimentos relacionados à conjuntura atual; o maior tempo de exposição às possíveis violências domésticas; o medo do adoecimento e da morte; o agravamento das expressões da questão social; as mudanças na rotina e na convivência social e comunitária decorrentes dos necessários protocolos de biossegurança editados pelas autoridades governamentais e sanitárias são alguns dos elementos presentes em um cenário de pandemia e que podem impactar na saúde mental das pessoas ${ }^{67}$.

Estudos feitos com populações envolvidas em uma ameaça que gera medo ou terror, identificaram que mais de $80 \%$ das pessoas desenvolvem sintomas de medo ou pânico. Pode-se considerar ainda que entre um terço e metade da população exposta a uma epidemia de grande magnitude tem a probabilidade de sofrer alguma manifestação psicopatológica ${ }^{8}$. Algumas das reações esperadas em uma epidemia podem incluir o medo de adoecer e de morrer; sentimentos de desamparo, tédio, solidão e depressão devido ao isolamento; medo de demissão e de perder os meios de subsistência; medo de perder familiares e amigos; assim como o medo de infectar outras pessoas ${ }^{9}$.

O impacto será percebido conforme a pandemia evolui e após o término dela, diante das consequências econômicas, sociais e culturais geradas ou agravadas. As pesquisas que avaliaram as consequências psicológicas da quarentena em epidemias recentes identificaram a presença de efeitos negativos tais como irritabilidade, humor deprimido, confusão, raiva, insônia, exaustão emocional e sintomas de estresse pós 
traumático ${ }^{10}$. No decorrer da quarentena os fatores que mais impactaram negativamente foram a duração do distanciamento social imposto, o medo de infecção, a frustração e o tédio, as informações inadequadas acerca da situação, bem como a precariedade do acesso a suprimentos básicos ${ }^{10}$. Já após a quarentena os problemas financeiros e o estigma especialmente relacionado aos profissionais de saúde - se destacaram como principais estressores ${ }^{11}$. Os referidos estudos envolveram medidas de quarentena ocorridas em 10 países durante as seguintes emergências em saúde: o surto de SARS e de Ebola, a pandemia de influenza H1N1 de 2009 e 2010, a MERS e a influenza equina ${ }^{11}$.

Como já mencionado anteriormente, o isolamento social foi uma das medidas operacionalizadas para diminuir o número de óbitos, em decorrência da pandemia de COVID-19. Ocasionando o fechamento de escolas, universidades, restaurantes, shoppings, cancelamento de eventos que gerem aglomeração de pessoas, dentre outras. Uma oportunidade de ressignificar a rotina, lançando mão de novas estratégias que adaptem a realidade imposta ou uma maneira passageira de enfrentamento dos problemas advindos do confinamento. Diante desse cenário, a OMS, preocupada com as consequências futuras, desenvolveu um guia relacionando os cuidados em saúde mental em meio a pandemia. Este guia vem sendo utilizado como referência pelos estados e municípios do Brasil, de modo a orientar os profissionais da saúde no desenho de linhas de cuidado em saúde mental ${ }^{12}$.

Portanto, o isolamento social pode impactar negativamente na saúde mental da população brasileira. É necessário que as autoridades ofereçam suporte adequado aos trabalhadores, usuários e gestores, para que possam difundir estratégias factíveis de prevenção de agravos e promoção da saúde mental ${ }^{10}$. Além do desenvolvimento de ações e serviços de saúde mental compatíveis com as singularidades sociais, econômicas e culturais de cada estado e do Distrito Federal. Ademais, torna-se importante a utilização 
de ferramentas de monitoramento e a avaliação para a adequação das estratégias de enfrentamento, tendo em vista o excesso de informações desconectadas ${ }^{13}$.

Diante disso, este estudo tem como intuito saber de que forma as normativas direcionadas aos cuidados em saúde mental estão sendo divulgadas para a população, para os profissionais de saúde e para os gestores, bem como acerca das estratégias de enfrentamento recomendadas por estes documentos em circunstâncias da pandemia de COVID-19.

\section{MÉTODOS}

Trata-se de uma revisão de narrativa com a proposta de trazer à tona as orientações sobre enfrentamentos e estratégias estabelecidas por normativas governamentais, acerca da saúde mental durante a pandemia por COVID-19. Caracterizada por ser um método intuitivo de mapeamento na literatura e em outras fontes de informação direta, a revisão de narrativa permite levantar dados investigativos ampliados sobre o tema proposto, tendo em vista o "estado da arte" acerca dos referenciais teóricos e suas lacunas ${ }^{14} 1516$.

A intenção é desenvolver reflexões sobre as publicações governamentais voltadas aos cuidados de saúde mental da população, bem como acerca das orientações aos profissionais de saúde, no contexto da pandemia de COVID-19, e assim, servir de apoio e esclarecer as informações de saúde mental aos trabalhadores e usuários da saúde. Nesse sentido, foram realizadas sucessivas consultas em bancos de dados, portais de pesquisa, sites de organizações relacionados à saúde pública e sites oficiais, entre os meses de março e abril de 2020, com o uso dos seguintes descritores: "coronavírus e saúde mental"; "saúde mental COVID-19"; "saúde mental e isolamento"; "isolamento social e saúde 
mental"; "psicossocial"; "nota técnica COVID-19 e saúde mental" e "novo coronavírus e saúde mental”. E, assim, realizar uma varredura por meio destes descritores.

\section{RESULTADOS E DISCUSSÕES}

No contexto de pandemia é imprescindível que as autoridades estejam alinhadas sobre as informações que poderão ser prestadas à comunidade. E, ainda, que elas possam preencher lacunas de processos de cuidados com o alcance a população, profissionais e gestores da saúde da saúde mental, que tem se tornado cada vez mais latente aos diversos problemas que se apresentam pelo isolamento social ${ }^{11}$. Foram encontrados 9 normativas pelos meios de procura utilizados na pesquisa.

Na região Centro-oeste, o estado do Goiás confeccionou um "guia de cuidado da saúde mental na pandemia da COVID-19 e isolamento social”, o qual traz orientações à população de modo geral. Inicialmente apresenta informações sobre o isolamento social e os enfrentamentos que acometem a rotina, caminhando para propostas e estratégias diante do contexto. Problematiza quanto às dificuldades e recursos que possam confrontar os danos advindos do isolamento social. A readaptação é colocada como forma de viabilizar mudanças significativas no cotidiano. É dividido em sete propostas imperativas, como: evite excesso de informações desnecessárias; evite pensamentos vitimistas; evite solidão como percepção de abandono; evite ficar ocioso; reoriente o seu trabalho; envolva a família em atividades domésticas; ajude o próximo ${ }^{17}$.

O manual se caracteriza como ferramenta de fácil compreensão e acesso à população, no entanto, responsabiliza os usuários na inovação e criação de ferramentas para o seu próprio cuidado e com as outras pessoas. A cartilha estabelece condutas firmas no imediatismo não colocando estratégias de enfrentamentos longitudinais como é 
sugerido no documento da Fiocruz $(2020)^{17}$, que estabelece condutas de gestão e assistências no trato da COVID-19. Como por exemplo, "impacto traumático do COVID19", dimensionar formas de enfrentamento à doença, e utilização das esferas sociais e culturais com objetivos concretos, com ações que visam a prevenção e promoção ${ }^{10} 17$.

No Distrito Federal, a Secretaria de Estado de Saúde (SESDF), publicou uma nota técnica informativa "a saúde mental dos profissionais de saúde em meio à pandemia COVID-19”, objetivando orientações de cuidados da saúde mental dos profissionais que estão na ponta. Com destaque para a "estigmatização por trabalhar com o paciente com COVID-19”, hipervigilância, necessidade de adaptação a novos arranjos de trabalho, luto pela perda de colegas de trabalho, dentre outros. Como estratégia, orienta: reservar tempo para a reflexão e descanso, alimentação saudável, evitar o uso de álcool e outras drogas, práticas de leitura e atividade física, autoconhecimento etc ${ }^{18}$.

A nota ainda traz recomendações aos trabalhadores para a condução dos cuidados como: "o contágio emocional", que está relacionado à reação quanto ao ambiente e contexto cultural, dentre elas: as reações agudas de estresse e transtorno de ajustamento são os transtornos mais identificados em estresse coletivo; o transtorno de estresse póstraumático, que costuma aparecer em decorrência de um evento específico; as equipes também devem estar atentas quando os quadros de transtornos depressivos e de ansiedade persistem; o luto, com a oportunidade de prestar suporte para a elaboração do processo de perda. Delirium, flutuações de consciência e desorientação. Tendo em vista que chama a atenção para o risco de patologização da vida, tratando os processos de enfrentamentos das pessoas, como transtornos mentais. Pois o fechamento do diagnóstico da pessoa em sofrimento psíquico é sobremaneiramente complexo, é importante observar a pessoa e o contexto que ela está inserida 181920 . 
Além disso, a nota técnica informativa orienta quanto aos casos de transtorno de bipolaridade e esquizofrenia, para que não interrompa o tratamento, e disponibilidade de prescrição dos medicamentos por algum tempo, sendo essencial trabalhar dentro de um plano de segurança e comunicação em rede dos serviços de saúde mental. E instruir sobre as consequências ao longo prazo, como o restabelecimento da rotina dos equipamentos de saúde mental, com agendas mais flexíveis e voltadas a questões trazidas dos usuários, para que que construa uma carteira de serviços ancoradas as necessidades, pós-pandemia, para a população. A nota informativa, segue as orientações do Ministério da Saúde (MS), que na nota técnica $\mathrm{N}^{\circ} 12 / 2020-\mathrm{CGMAD/}$ DAPES/ SAPS/ MS, trata sobre as recomendações à rede de atenção psicossocial (RAPS) sobre estratégias de organização no contexto da infecção da COVID-19 causado pelo novo coronavírus (sars-cov-2) ${ }^{21}$.

O estado do Mato Grosso do Sul, comunica a população no seu portal de notícias da Secretaria de Saúde, com o seguinte título "Coronavírus: saúde mental também precisa de atenção e cuidados”. O site evidência, logo de primeira mão, a necessidade dos cuidados com a higiene, com ênfase na questão da saúde mental. Fundamentados na OMS, e por meio de consultoria de um especialista, fazem um recorte das principais medidas e cuidados recomendados à população em geral. Explicando sobre os cuidados com pessoas vulneráveis, como também instruções de canais de ajuda no domicílio. E fechando com dicas para o enfrentamento do isolamento social. $\mathrm{O}$ que atende às orientações da OMS e do MS, mas que não se compromete com fortalecimento da rede psicossocial e em instrumentalizar serviços de saúde mental a população em geral ${ }^{22} 23$.

Em São Paulo, a partir da nota técnica 2, de 25/03/2020, que dispõe sobre: Atuação e Funcionamento dos Serviços de Saúde Mental que compõem a Rede de Atenção Psicossocial quanto aos cuidados em relação ao coronavírus - COVID-19, face a declaração da OMS (2020) $)^{22}$, estabelece um plano organizativo para todos os serviços 
de saúde mental. Iniciando pela reorganização do fluxo de oferta, cuidados sanitários, observância dos cuidados de higienização. Orienta, também sobre os dispositivos de proteção para os usuários, como também a interrupção de todas as atividades coletivas, sendo substituída por individuais e em locais ventilados. Trata sobre os atendimentos a distância em saúde mental. Incentiva o acompanhamento e monitoramento, por telefone, de usuários com vulnerabilidade, principalmente os que se encontram nos hospitais psiquiátricos. Sugere a disposição de cartazes nas entradas dos equipamentos, com informações sobre os sintomas da doença ${ }^{24}$.

Discrimina sobre as Residências Terapêuticas em que os trabalhadores devem prestar informações sobre os protocolos de higienização e que o gestor deve garantir acesso aos insumos, e a implantação das medidas de isolamento social. Orienta que os trabalhadores da saúde mental devem cuidar para evitar stress e ansiedade. Finaliza orientando todos os trabalhadores a leitura na íntegra do documento sobre "cuidados em saúde mental para a população e para os serviços de saúde em geral - OMS”, pois identifica o contexto de pandemia e consequentemente do isolamento social um ambiente favorável ao desenvolvimento de inquietudes para a população em geral e da população em sofrimento mental ${ }^{24}$. Os cuidados e reorientação dos equipamentos são ações essenciais para a qualidade e eficácia do trabalho, e São Paulo teve recomendações importantes para os gestores e trabalhadores.

Pelo governo de Minas Gerais foi construído uma cartilha que organizou as principais informações sobre a atenção psicossocial e a situação de pandemia da COVID19. As orientações que tange "saúde mental e atenção psicossocial frente a pandemia do coronavírus", seccionada em 3 partes. A primeira diz respeito a população em geral, comunicando sobre o entendimento de empatia, para que nesse novo cenário não ocorram discriminações e, consequentemente, não criar estigma e nem consumir muita informação 
em excesso. Cuidados com próximo e principalmente aqueles que estão em risco. Que o contato social deve ser substituído por virtual, o compartilhamento de histórias e informações positivas. Orienta-se sobre a organização pessoal e profissional, a proteção aos idosos e as crianças. A segunda parte do informativo diz respeito aos trabalhadores da saúde, onde se destacam: empatia, informação qualificada, respeito as diferenças, descanso e autocuidado e contato social. A terceira e última, são recomendações aos gestores de saúde, como: qualidade na informação, criação de um sistema de apoio e troca de saberes entre os trabalhadores, apoio social e o suporte de insumos ${ }^{25}$. Expressa cuidados com a população em geral, trabalhadores da saúde e gestores, estando em consonância com um documento da Fiocruz que também tem os cuidados divididos entre esses atores. Com destaque para um espaço de troca de saberes e autocuidado entre os trabalhadores ${ }^{26}$.

Na região sul, a Secretaria de Saúde do Paraná destaca a importância da mobilização dos profissionais de saúde da atenção psicossocial no contexto de pandemia, reconhecendo a fragilidade emocional pelas quais passam todos aqueles que já são vulneráveis pela sua própria condição de sofrimento mental. Traz como recomendações para a população: manter a rotina e horários de sono, alimentação saudável, de organização da casa, realizar atividades físicas e buscar formas de relaxamento, como conversar com pessoas próximas. Destaca ainda que o profissional de saúde mental deve oferecer suporte e apoio aos trabalhadores de saúde que estão atuando na linha de frente 27. No entanto, vale destacar que todos os profissionais de saúde devem ser capacitados para realizar ações de promoção e educação em saúde mental, de modo que todos possam estar aptos a oferecer os cuidados de saúde mental, conforme documento desenvolvido pela Fundação Oswaldo Cruz (Fiocruz) ${ }^{10}$, que orienta os gestores sobre os cuidado em saúde mental durante a pandemia ${ }^{10}$. 
Já na região nordeste, pode-se destacar a cartilha formulada pela Secretaria de Saúde do Estado do Ceará, direcionada aos profissionais de saúde que atuam na RAPS. Intitulada Coronavírus (COVID-19) - Cuidados em Saúde Mental, a cartilha, de início, estimula os trabalhadores da RAPS a prestar orientação e suporte aos “colegas que estão em ações de enfrentamento ao coronavírus (Covid-19)”. Pode-se inferir que tais colegas são os profissionais de saúde que atuam diretamente com casos confirmados de COVID19. No entanto, é válido ressaltar o caráter nebuloso de tal afirmativa, tendo em vista as diversas áreas que estão envolvidas no enfrentamento à pandemia. O documento sugere o envolvimento da Atenção Especializada, da Vigilância Sanitária e da Atenção Primária em ações conjuntas com o intuito de informar a população acerca de "profilaxia, prevenção e cuidados em saúde mental". E por fim, propõe orientações a serem prestadas durante a assistência aos usuários dos serviços ${ }^{28 .}$

Algumas dessas orientações são pontuais como buscar informações em fontes seguras e atentar-se para o excesso de notícias; umas simplistas e pouco elucidativas: orientar sobre práticas de atividade física em ambiente aberto; enquanto outras se destacam ao mencionar o plano terapêutico singular (PTS), o qual deve explorar especialmente o ambiente doméstico, tendo em vista as medidas de biossegurança que impactam na reorganização dos fluxos dos serviços e nas rotinas das pessoas. A referida cartilha apresenta alguns elementos - mais precisamente no que tange às fontes de informações e à necessidade de adaptação do PTS - consonantes à nota técnica editada pelo Ministério da Saúde, a qual enuncia recomendações à RAPS sobre estratégias de organização no contexto da pandemia de COVID-19 ${ }^{21}$.

A publicação encontrada no estado de Alagoas diz respeito à matéria no site da Secretaria de Estado da Saúde elaborada pelo setor de Supervisão de Atenção Psicossocial. O texto enfatiza a importância das medidas restritivas de circulação como 
fator primordial para evitar as mortes por COVID-19 e direciona à população orientações de cuidados, objetivando a manutenção da saúde mental em contexto de isolamento social. A intenção pode ser sintetizada pelo trecho "[...] fique em casa! Mas fique bem.” 29. As principais sugestões giram em torno do estabelecimento de rotinas de estudo e de trabalho, com foco na produtividade; da execução de atividades que gerem prazer; do controle quanto ao tempo de uso de redes sociais, à quantidade e à qualidade das informações consumidas, as quais devem advir de fontes oficiais; da prática de atividade física, que pode, inclusive, envolver outras pessoas que residem na mesma casa; da prática da resiliência em casos de dificuldades de convivência com membros do núcleo familiar; da prática da espiritualidade na residência, em detrimento de locais públicos; bem como de evitar o consumo excessivo de bebidas alcoólicas, citando a dependência como possível resultado desse uso ${ }^{29}$.

É interessante a publicação considerar que as relações podem ser conflituosas, no entanto, o ambiente doméstico também tem a possibilidade de ser um local inseguro, de ocorrência de violências. A "calma, paciência, tolerância e respeito" e a prática da “resiliência" sugeridos não cabem em um contexto de violação de direitos ${ }^{29}$. Dados apontam que durante a pandemia de COVID-19 o número de casos de violência doméstica na China triplicou, estima-se que no Brasil o crescimento foi de até $50 \%$, enquanto a França apresentou aumento de $30 \%$. A Itália também registrou aumento das denúncias de violência doméstica ${ }^{30}$. Tal realidade não pode ser descolada das orientações em saúde mental, tendo em vista que a presença da referida violência gera sérias consequências psicológicas em quem as sofre ${ }^{31}$.

Outra normativa encontrada na região nordeste se refere à publicada pela Secretaria de Estado da Saúde do Piauí, por meio da Gerência de Atenção à Saúde Mental. O documento denominado "Instrutivo sobre atenção psicossocial em face da 
pandemia do novo coronavírus" apresenta orientações técnicas direcionadas aos profissionais e aos gestores. Apesar de logo em seguida apresentar os possíveis transtornos mentais decorrentes de uma situação de pandemia, chama a atenção para a não patologização de todas as reações esperadas nesse período, indo ao encontro do que é mencionado em documento elaborado pela Fiocruz ${ }^{26}$. A publicação governamental reforça a importância do fortalecimento das políticas públicas voltadas ao cuidado da saúde mental da população e da utilização de estratégias consonantes com as recomendações do Ministério da Saúde. Cita como estratégia de resposta às demandas de saúde mental a inserção de profissionais da RAPS nos Comitês de Operações de Emergências em Saúde existentes nos municípios. Outro ponto a ser destacado é o que salienta sobre a necessidade de manutenção dos atendimentos de urgência a crise em saúde mental nos serviços de atenção especializada, em consonância com o recomendado pela nota técnica do Ministério da Saúde que dispõe sobre a organização da RAPS frente à pandemia de COVID-19 2132 .

O referido instrutivo finaliza sinalizando a importância dos gestores e coordenadores das equipes de saúde atentarem-se para a saúde mental dos trabalhadores, devendo considerar a qualidade das informações prestadas à equipe, a criação de ambiente que fomente o apoio mútuo entre os profissionais, assim como a formação de sistema de apoio e troca de saberes entre colegas ao reunir trabalhadores mais e menos experientes na mesma equipe. Também orienta os gestores em relação à garantia do fornecimento essencial de insumos de saúde para todos os níveis de cuidados, bem como às medidas de prevenção e controle no âmbito do trabalho, no que se refere aos equipamentos de proteção individual (EPI) ${ }^{32}$. Nota-se que estado do Piauí direciona tal sistematização de ações e procedimentos referentes à saúde mental de forma que permite subsidiar adequadamente as ações de gestores e trabalhadores. 


\section{CONSIDERAÇÕES FINAIS}

Sabe-se que este período de pandemia, em especial a quarentena, tende a aumentar de forma expressiva o sofrimento mental na população em geral, em decorrência do isolamento social e limitação da comunicação interpessoal. Além disso, os profissionais de saúde que estão na linha de frente possuem alto risco de desenvolver tais problemas, necessitando de intervenções que possam apoiá-los neste momento. Tendo em vista que o impacto psicossocial estará presente nas diferentes fases da pandemia - inclusive após o seu fim -, torna-se necessário estruturar planos de ações amplos, que visem integrar estratégias de intervenções para a população em geral.

Tais estratégias devem envolver as diferentes necessidades da população, de modo a alcançar as esferas sociais, culturais, religiosas e artísticas, as quais contribuem para a manutenção da saúde mental. Além disso, cada estado precisa avaliar as particularidades do seu território, de modo a desenvolver planos de cuidado em saúde mental que estejam em consonância com as recomendações da Organização Mundial de Saúde e do Ministério da Saúde.

Vale destacar que, as universidades possuem uma grande produção científica, o que contribui para a tomada de decisão da gestão pública e dos serviços de saúde. Além disso, há diversos projetos que são realizados a partir de uma integração entre ensinoserviço-comunidade, o que possibilita uma articulação com os projetos desenvolvidos pelas secretarias de saúde dos estados e municípios, fortalecendo cada vez mais os planos de ações, gerando um impacto significativo para a sociedade. De antemão, reconhecemos que o entendimento sobre os arranjos e estratégias que contribuem para o enfrentamento da pandemia se converte numa sintonia de propostas e ideias que devem ser contextualizadas culturalmente, socialmente e economicamente. 


\section{REFERÊNCIAS}

1. Rothan HA, Byrareddy SN. The epidemiology and pathogenesis of coronavirus disease (COVID-19) outbreak. J Autoimmun. 2020;109:102433. doi:10.1016/j.jaut.2020.102433.

2.Kritski, et al. Nota Técnica de pesquisadores da Universidade Federal do Rio de Janeiro (UFRJ), Universidade de São Paulo (USP) e Universidade de Brasília (UnB) 25 de março 2020.

3.Ministério da Saúde. Brasil registra 40.581 casos de coronavírus e 2.575 mortes. Disponível em: < https://www.saude.gov.br/noticias/agencia-saude/46757-brasilregistra-40-581-casos-de-coronavirus-e-2-575-mortes >. Acesso em: 20 de abril de 2020.

4.Rodrigues C, Barros, H. Da emergência de um novo vírus humano à disseminação global de uma nova doença - Doença por Coronavírus 2019 (COVID-19). Secretaria da Saúde do Estado do Ceará - SESA/CE. Coronavírus (COVID-19) - Cuidados em Saúde Mental. Disponível em: <https://drive.google.com/file/d/1DLMBZyLX3b0CN3YF0krQJOmdI4ZcZ7d/view>. Acesso em 21 de abril de 2020.

5.Armitage R, Nellums, L. B. COVID-19 and the consequences of isolating the elderly. The Lancet Public Health; 2020: 5, e-256. Disponível em: < 
https://www.thelancet.com/action/showPdf?pii=S2468-2667\%2820\%2930061-X>

Acesso em 21 de abril de 2020.

6. Ornell F, et al. Pandemia de medo e CoVid-19: impacto na saúde mental e PossíVeis estratégias. Debates in psychiatry - Ahead of print 2020.

7. Afonso P. The Impact of the COVID-19 Pandemic on Mental Health. Acta Médica Portuguesa, [S.1.], v. 33, n. 13, apr. 2020.

8. OPAS/OMS - Organização Pan-Americana da Saúde/OMS. (2019). Proteção da Saúde Mental em Situações de Epidemias. Disponível em: <https://www.paho.org/hq/dmdocuments/2009/Protecao-da-Saude-Mental-emSituaciones-de-Epidemias--Portugues.pdf >. Acesso em: 18 de abril de 2020.

9. IASC - Inter-Agency Standing Committee. (2020). Como lidar com os aspectos psicossociais e de saúde mental referentes ao surto de COVID-19. Versão 1.5, março 2020.

10. Fiocruz. Saúde mental e Atenção Psicossocial na Pandemia COVI-19. $\begin{array}{lllll}\text { Recomendações } & \text { para } & \text { gestores. } & \text { Disponível em }\end{array}$ <https://www.unasus.gov.br/especial/covid19/pdf/110> Acesso em 23 de abril de 2020.

11. Brooks SK; et al. The psychological impact of quarantine and how to reduce it: rapid review of the evidence. The Lancet, 2020.

12. Schmidt B; et al. Crepaldi, M. A.; Bolze, S. D. A.; Neiva-Silva, L.; Demenech, L. M. Impactos na Saúde Mental e Intervenções Psicológicas Diante da Pandemia do Novo Coronavírus (COVID-19). Estudos de Psicologia, Campinas, 2020. 
13. Chevance, A. et al. Assurer les soins aux patients souffrant de troubles psychiques en France pendant l'épidémie à SARS-CoV-2. L'Encéphale, 2020.

14. Toledo, Juliane Alvarez de; Rodrigues, Marisa Cosenza. Teoria da mente em adultos: uma revisão narrativa da literatura. Bol. - Acad. Paul. Psicol., São Paulo, v. 37, n. 92, p. 139-156, jan. 2017.

15.Nascimento Priscila, et al. Depressão e fragilidade na velhice: uma revisão narrativa das publicações de 2008-2018. Interface (Botucatu), Botucatu, v. 23, e180609, 2019.

16. Chaves L, et al. Desabastecimento de medicamentos na literatura científica da saúde: uma revisão narrativa. Physis, Rio de Janeiro, v. 29, n. 1, e290107, 2019.

17. Secretaria de Estado de Saúde de Goiás. Guia de Cuidado da Saúde Mental na

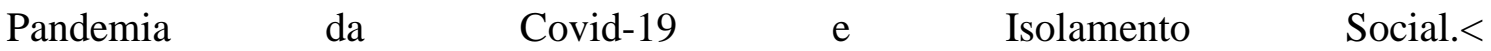
https://www.saude.go.gov.br/files/banner_coronavirus/manual_saudementalcoronavirus.pdf $>$ Acessado dia 16 de abril de 2020.

18. Secretaria de Estado de Saúde do Distrito Federal< http://www.saude.df.gov.br/wpconteudo/uploads/2018/03/Nota-Informativa-A-Sa\%C3\%BAde-Mental-e-a-Pandemiade-COVID-19-impactos-e-orienta\%C3\%A7\%C3\%B5es-para-profissionais-desa\%C3\%BAde.pdf> acessado 23 abril de 2020

19. Martinhago F, Caponi S. Controvérsias sobre o uso do DSM para diagnósticos de transtornos mentais. Physis, Rio de Janeiro, v. 29, n. 2, e290213, 2019.

20. Amarante, P. (1996). O homem e a serpente: outras histórias para a loucura e a psiquiatria. Rio de Janeiro: FIOCRUZ. ISBN: 85-85676-89-2.

21. BRASIL. Ministério da Saúde. Nota técnica n 12/2020-CGMAD/ DAPES/ SAPS/ MS. 
<http://189.28.128.100/dab/docs/portaldab/documentos/notatecnica122020CGMADDA PESSAPSMS02abr2020COVID-19.pdf>. Acesso em 24 de abril de 2020.

22- WHO. Mental health and psychosocial considerations during COVID-19 outbreak. March, 2020. Disponível em < https://www.who.int/docs/defaultsource/coronaviruse/mental-health-considerations.pdf >. Acesso em 23 de abril de 2020.

23. Secretaria de Estado de Saúde do Mato Grosso do Sul. Disponível em <http://www.ms.gov.br/coronavirus-saude-mental-tambem-precisa-de-atencao-ecuidados/> Acessado 17 de abril de 2020.

24.Nota Técnica 2, de 25 de março de 2020 - Coordenadoria de Controle de Doenças: Atuação e Funcionamento dos Serviços de Saúde Mental que compõem a Rede de Atenção Psicossocial quanto aos cuidados em relação ao coronavírus - COVID19<http://www.cosemssp.org.br/wp-content/uploads/2020/03/Nota-Te\%CC\%81cnica2-de-25de-marc\%CC\%A7o-de-2020.pdf> acessado 10 de abril de 2020.

25. Secretaria de Estado de Saúde de Minas Gerais. Saúde e Atenção Psicossocial frente à pandemia do coronavírus. Disponível em <http://www.cosemssp.org.br/wpcontent/uploads/2020/03/Cartilha-Sa\%C3\%BAde-Mental-e-Coronav\%C3\%ADrus.pdf> acessado dia 23 de abril de 2020.

26 Fiocruz - Recomendações Gerais- Saúde mental e atenção pscisossocial na pandemia COVID-19. Disponível em < https://www.unasus.gov.br/especial/covid19/pdf/110> Acessado em 20 de Abril de 2020.

27. Secretaria de Estado de Saúde do Paraná. Saúde Mental. Disponível em: < http://www.saude.pr.gov.br/arquivos/File/NO_15_SAUDE_MENTAL.pdf>. Acesso em 17 de abril de 2020. 
28. Secretaria de Estado de Saúde do Ceará. Coronavírus (COVID-19) - Cuidados em Saúde Mental. Disponível em: <https://drive.google.com/file/d/1DLMBZyLX3b0CN3YF0krQJOmdI4ZcZ7d/view>. Acesso em 21 de abril de 2020.

29. Secretaria de Estado da Saúde de Alagoas - SESAU/AL. Saiba como cuidar da saúde mental durante isolamento pela Covid-19. Disponível em: <http://www.saude.al.gov.br/2020/04/01/saiba-como-cuidar-da-saude-mental-duranteisolamento/>. Acesso em 20 de abril de 2020.

30. Campbell, Andrew M. An increasing risk of family violence during the Covid-19 pandemic: Strengthening community collaborations to save lives. Forensic Science International: Reports. v. 2, 2020.

31. Organização Mundial da Saúde (OMS). (2005). Estudio multipaís de la OMS sobre salud de la mujer y violencia doméstica contra la mujer: primeros resultados sobre prevalencia, eventos relativos a la salud y respuestas de las mujeres a dicha violencia. Resumen del Informe. Genebra: Organização Mundial da Saúde. Disponível em: <https://apps.who.int/iris/handle/10665/43390>. Acesso em: 25 de abril de 2020.

32. Secretaria de Estado da Saúde do Piauí - SESAPI. Instrutivo sobre atenção psicossocial em face da pandemia do novo coronavírus. Disponível em: <http://www.saude.pi.gov.br/uploads/warning_document/file/510/Instrutivo_sobre_aten \%C3\%A7\%C3\%A3o_psicossocial_em_face_da_pandemia_do_novo_coronav\%C3\%A Drus_PDF.pdf $>$. Acesso em 25 de abril de 2020. 
